\title{
IMAGING AND CHARACTERIZATION OF $y$ PRECIPITATES IN NICKEL-BASED SUPERALLOYS
}

\author{
Peter Sarosi ${ }^{1}$, Gopal Viswanathan ${ }^{1}$, Deborah Whitis ${ }^{2}$ and Michael Mills ${ }^{1}$ \\ ${ }^{1}$ Department of Materials Science and Engineering \\ The Ohio State University, Columbus, $\mathrm{OH}$ \\ ${ }^{2}$ Materials and Processes Engineering Department \\ GE Aircraft Engines, Cincinnati, OH
}

Keywords: EFTEM, Nickel-based superalloys, volume fraction, TEM

\begin{abstract}
Computer models are being increasingly used to reduce the cost of microstructure and alloy development and accelerate insertion of materials (AIM). In the case of "real" multi-component engineering nickel-based superalloys, initial studies from computer models predict extremely high sensitivities of creep and tensile behavior to size (d) and volume fraction (Vf) of fine $\gamma$ precipitates. However, predictions are currently based on input data ( $\mathrm{Vf}$ and $\mathrm{d}$ ) derived entirely from either estimated and/or severely limited experimental datasets because of the precipitate's small size. Therefore, in order to (1) confirm the validity of model's initial predictions and (2) consequently improve upon its predictive capabilities, it is necessary to acquire an extensive, empirical dataset to replace these unqualified input datasets. Consequently, we investigated a number of imaging techniques available in a Tecnai F-20 FEG/TEM and selected the technique which best enabled rapid and extensive acquisition of these datasets using the engineering alloy, Rene '88 DT. The EFTEM technique was found to be the most appropriate method for imaging fine $\gamma$ precipitates while further investigation showed that the $\mathrm{Cr} \mathrm{M}$-edge, in comparison with other ionization-edges provided the best images based largely on contrast-to-noise ratio. Imaging of the $\mathrm{Cr}$ M-edge elemental maps were further improved by investigating the effects of microscope parameters, imaging filter parameters and analysis of the experimental electron energy loss spectra obtained from this alloy. In addition, a novel technique to determine the volume fraction of the fine $\gamma$ precipitates without the need to determine the absolute thickness of the TEM foil is proposed.
\end{abstract}

\section{Introduction}

The excellent creep, tensile, fatigue and crack growth properties of nickel-based superalloys have made them the favored, hightemperature structural material used in the turbine section of jet engines. However, higher operating temperatures needed to increase engine efficiency degrade the superalloy's mechanical properties, and thus reduce the component's service life. This has led to the development of many proprietary, component-specific alloys, including Rene 88 DT [1] an alloy currently used in disk components. In the case of Rene 88 DT, microstructural changes that occur upon ageing and during service contribute strongly to the degradation of the mechanical properties - although the precise deformation mechanisms affecting creep and tensile behavior yet to be fully determined.
Recent work [1-3] has shown that microstructures consisting of multi-modal size distributions of $\gamma\left(\mathrm{Ni}_{3} \mathrm{Al}\right)$ precipitates in a $\gamma$ (FCC) matrix provide the best combination of tensile and creep strength. These multi-modal size distributions have been classified into three distinct groups - primary, secondary and tertiary $\gamma$ distributions. Primary $\gamma$ (usually the largest precipitate distribution) is formed during an initial subsolvus thermal process although Rene 88 DT is subjected to a super-solvus solution anneal and therefore contains no primary $\gamma^{\prime}$ [1]. The distinctly smaller secondary and tertiary $\gamma$ precipitates are formed by subsequent cooling and lower temperature isothermal ageing.

Quantitative microstructural data of these $\gamma$ precipitates are currently being incorporated into computer models for tensile and creep properties [4,5] - an increasingly cost-effective alternative to purely empirically-based models of behavior which require large, experimentally determined datasets to predict mechanical behavior of nickel-based superalloys. Importantly, in the case of "real" multi-component engineering nickel-based superalloys, initial studies from computer models predict extremely high sensitivities of creep and tensile behavior to size (d) and volume fraction (Vf) of fine $\gamma$ precipitates. However, these predictions are currently based on input data (Vf and d) derived entirely from estimated and severely limited experimental data of both secondary and tertiary $\gamma$ particularly for the latter due to their small size [1]. Therefore, in order to (1) confirm the validity of model's initial predictions and (2) consequently improve upon its predictive capabilities, it is necessary to acquire a preliminary set of experimentally determined microstructural data of both secondary and tertiary $\gamma$ to replace the initial and inadequate input datasets.

The size distributions and volume fractions of secondary and tertiary $\gamma$ are usually determined directly from either crosssectional 2D images, usually from secondary electron micrographs of slightly etched surfaces taken on the SEM, or from projected areal fractions of the microstructure from the $3 \mathrm{D}$ volume in a TEM foil. Previous work has shown that secondary $\gamma$ (average size $>30 \mathrm{~nm}$ ) can be imaged accurately and easily using SEM [1,6] but precise imaging of the smaller tertiary $\gamma$ using TEM has so far proved both difficult and time consuming [7]. The purpose of this study was to select an imaging technique which best enables rapid and extensive acquisition of microstructural datasets of small $\gamma$ precipitates from "real" multicomponent engineering alloys. 


\section{Experimental Procedures}

Rene 88 DT, which has a composition shown in Table i, was fabricated via powder metallurgy and samples were taken from the bore of a disk following various heat treatments. SEM samples were ground with $1200 \mathrm{SiC}$ paper, polished with colloidal silica and subsequently etched using a glyceregia solution [8]. Secondary Electron images were acquired on a Sirion FEG/SEM using an Ultra High Resolution (UHR) Through Lens Detector (TLD) operating at $10 \mathrm{kV}$.

Table i: Nominal composition of Rene 88 DT in atomic

\begin{tabular}{|c|c|c|c|c|c|c|c|}
\hline $\mathrm{Ni}$ & $\mathrm{Cr}$ & $\mathrm{Co}$ & $\mathrm{Al}$ & $\mathrm{Ti}$ & $\mathrm{Mo}$ & $\mathrm{W}$ & $\mathrm{C} / \mathrm{B} / \mathrm{Zr} / \mathrm{Nb}$ \\
\hline 56 & 18 & 13 & 4.5 & 4.5 & 2.4 & 1.3 & all $<0.5$ \\
\hline
\end{tabular}

Slices, $0.3 \mathrm{~mm}$ thick from the heat-treated samples were cut from the bulk material using an Accutom high-speed cutting saw and 3 $\mathrm{mm}$ precursor TEM discs were slurry drilled from slices. The TEM discs were ground to $100 \mathrm{~mm}$ using $1200 \mathrm{SiC}$ paper and an electron transparent area in the sample was formed by electropolishing in a Struers Tenupol-3 twin-jet electropolisher using an electrolyte containing $60 \%$ Methanol and $34 \%$ butylcellasove, $6 \%$ Perchloric $60 \%$ at $-40^{\circ} \mathrm{C}$ at an applied potential of $24 \mathrm{~V}$. A Tecnai-F20 FEG/TEM was used for all TEM imaging. High Angle Annular Dark Field (HAADF) images were acquired in Scanning Transmission Electron Microscopy (STEM) mode operating at $200 \mathrm{kV}$.

Energy Filtered Transmission Electron Microscope (EFTEM) images were acquired at $198 \mathrm{kV}$ using a Gatan Image Filter (GIF) with a slow-scan, YAG scintillator. The three-window method was employed using the power law function to subtract the background [9] using Digital Micrograph software.

\section{Results}

The large secondary $\gamma$ precipitates were imaged and subsequently characterized using SEM but the tertiary $\gamma$ precipitates were too small to image accurately in this manner. More suitable imaging techniques available in the TEM were then compared, based on a number of criteria: speed of image acquisition, image resolution, and strength of contrast (to enable automated computer image processing). The problem of determining volume fraction from the projected areal fraction of precipitates will be described later.

\section{$\underline{\text { STEM Imaging }}$}

Conventional and STEM Bright/Dark Field imaging were used but quickly discounted because the intensities of the superlattice reflections used to generate image contrast between $\gamma$ and the $\gamma$ matrix were very weak which made imaging very difficult. Fig. 1 shows a DF image acquired on the CCD detector. STEM imaging using the HAADF detector was also used to image tertiary $\gamma$ precipitates. Fig. 2 shows a typical HAADF image of a microstructure containing both secondary and tertiary $\gamma$. However, contrast generated from thickness variations in the foil caused by preferential etching of the matrix during electropolishing was also observed, offsetting and in some cases inverting the effect of Z-contrast. This can be seen in Fig. 3a, were both secondary $\gamma$ and more critically, the tertiary $\gamma$ appear either darker or lighter in comparison with the matrix which can therefore lead to underestimates of the projected areal fraction. It is likely that the brighter $\gamma$ precipitates are protruding from the foil, increasing locally the thickness of the foil - thus adding sufficient image intensity from the thickness component of STEM image to make the $\gamma$ appear brighter. Those precipitates

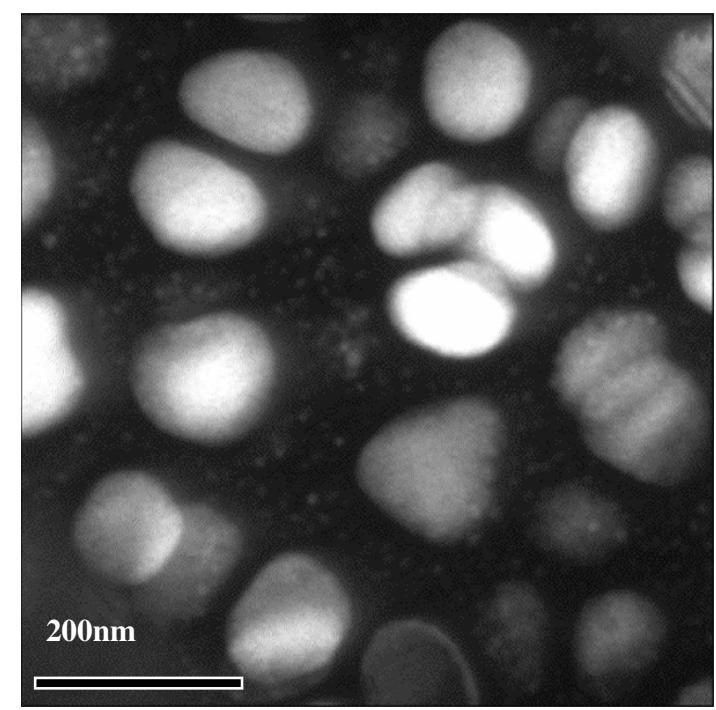

Figure 1: Dark Field image showing larger secondary $\gamma^{\prime}$ and some tertiary $\gamma^{\prime}$ from a sample cooled at $400^{\circ} \mathrm{F} / \mathrm{min}$

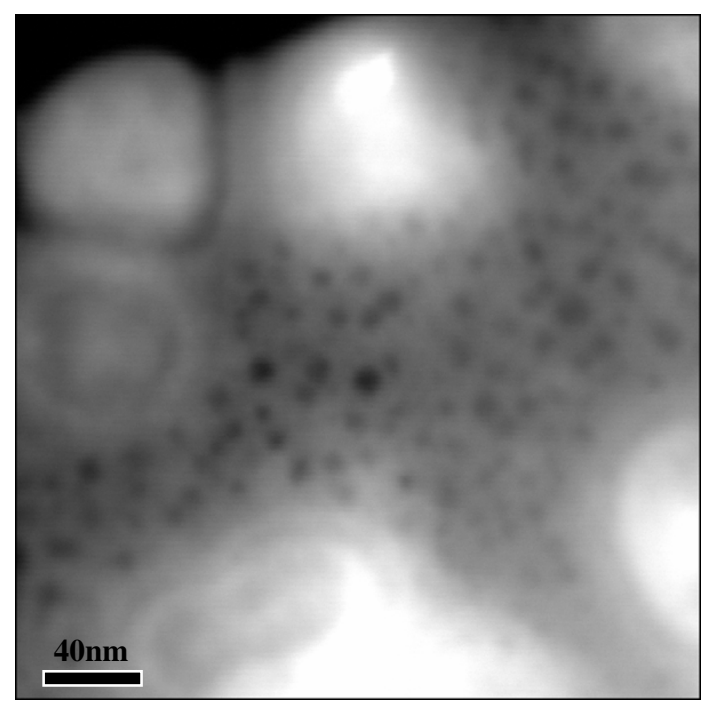

Figure 2: Z-contrast STEM image using a HAADF detector showing the bright secondary and finer, dark tertiary $\gamma^{\prime}$ precipitates.

which appear dark are fully embedded in the matrix and therefore do not add to the thickness contrast, leaving only Z-contrast to contribute to image intensity. Since the average atomic number is lower in $\gamma$ than in the matrix, the $\gamma$ appears darker in this case.

Figure 3: a) Z-contrast STEM image of tertiary $\gamma^{\prime}$ 

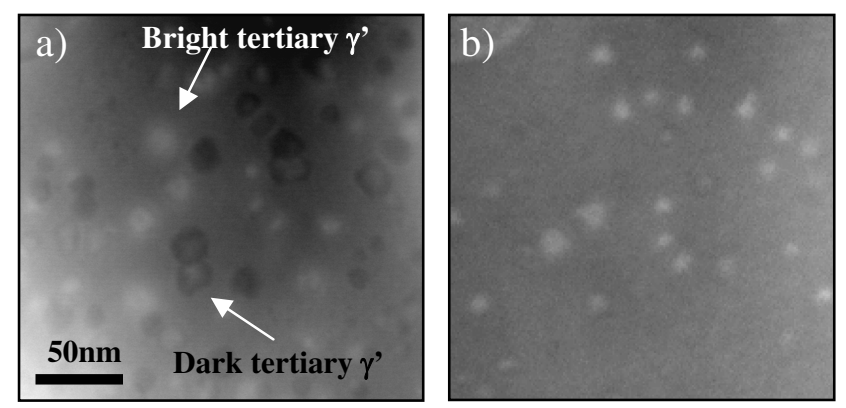

precipitates appearing darker and brighter than the matrix indicating mass and thickness contrast effects b) Secondary electron image taken in the SEM indicating that only those precipitates which protrude form the top of the TEM foil appear brighter in both STEM and SEM images.

The differences in elemental concentrations between $\gamma$ and $\gamma$ allowed elemental maps to be formed using the STEM mode coupled with EDX spectroscopy. Suitable alloying elements used for the elemental mapping were chosen based on the strength of partitioning shown in the line profile in Fig. 4. An elemental map requires an EDX spectrum to be collected for each pixel where each spectrum needs a 2 second acquisition time to ensure sufficient statistical data which for a standard 256x256 (low resolution) elemental map would require $\sim 1.5$ days - an inappropriately long acquisition time. The EDX map was therefore discounted for further use in this study.

\section{Energy Filtered Transmission Electron Microscopy (EFTEM)}

An energy filtered (EF) image is formed from a post-specimen electron beam that has undergone a specific energy loss due to a characteristic interaction of the electron beam with the sample. The tremendous advantage of EF imaging over STEM mapping is that highly spatially resolved images can be acquired over a large region of interest up to 1000 times faster than the acquisition of STEM elemental maps. This is because EF images are acquired in parallel (simultaneously) unlike the serially (sequentially) acquired STEM maps. In addition, chemical differences other than compositional can be used to generate EF images, unlike EDX spectroscopy which can only generate contrast from compositional information (variation).

EF imaging had already been used to image ternary nickel-based alloys [10], while Hatterstrand and Andren [11] used EF imaging to determine size and volume fraction of precipitates embedded in a matrix. Hofer et al [10] produced jump ratio images using $\mathrm{Cr}-\mathrm{L}_{2,3}$, $\mathrm{Ni}_{2,3}$ and $\mathrm{Ti} \mathrm{L}_{2,3}$ edges under rocking beam illumination, although the $\gamma$ precipitates were large enough $(\sim 30 \mathrm{~nm})$ to image with conventional SEM. Meanwhile, Hatterstrand and Andren

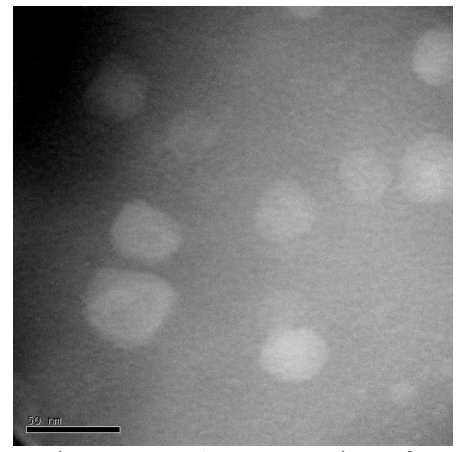

Figure 5: An example of a plasmon image with some contrast between $\gamma$ and $\gamma^{\prime}$, including the tertiary $\gamma^{\prime}$.
[11] had developed an equation to determine the size and volume

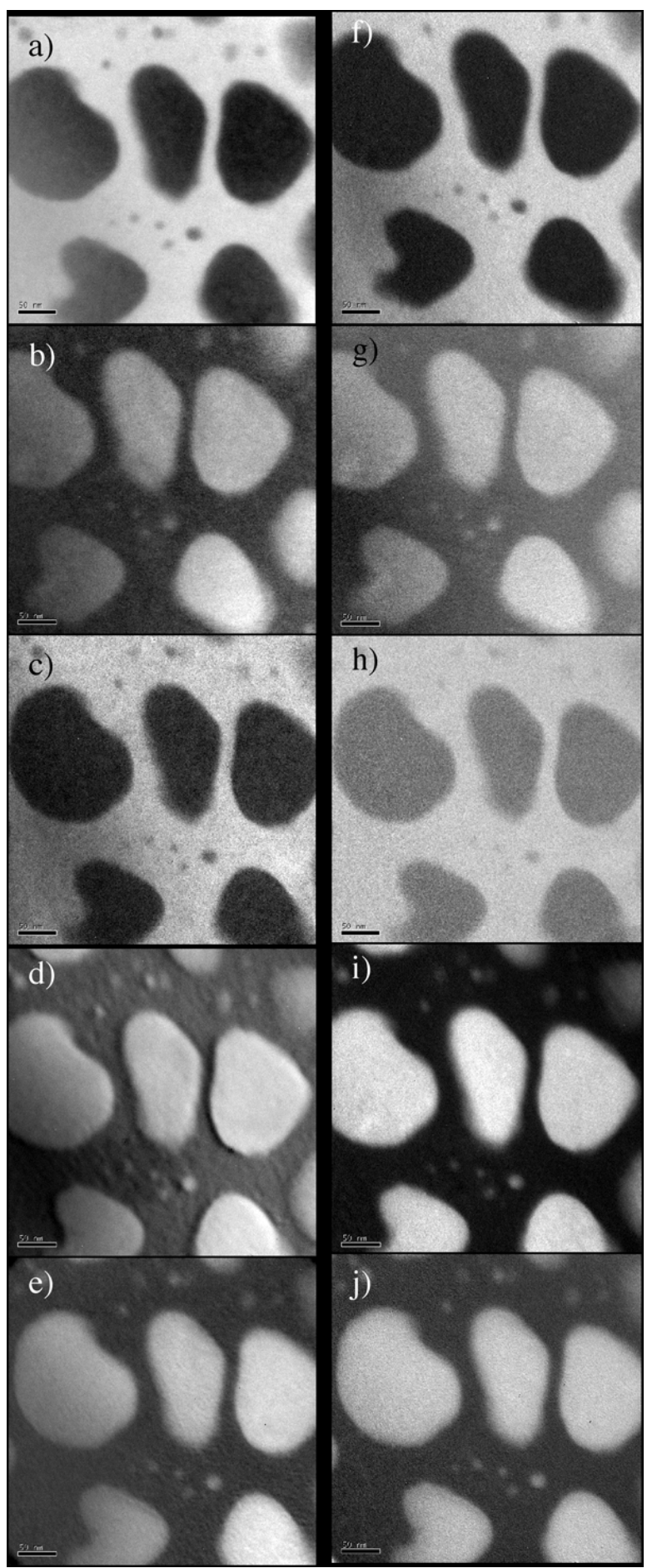

Figure 6: Elemental maps (a-e) and jump ratio images (f-j) images images using a,f) Cr L-edge b,g) Al Kedge, c,h) Co L-edge d,i) Ni L-edge e,j) Ti L-edge showing secondary and tertiary $\gamma^{\prime}$

fraction of spherical particles of uniform size, embedded in a matrix from projected 2D images of a finite volume. Hatterstrand and Andren's investigation involved the examination of niobium carbonitrides in stablilized stainless steel using EF images. However, both Hofer et al and Hatterstrand and Andren had imaged particles that were large enough such that thick foils $(\sim 100 \mathrm{~nm})$ could be used while still being able to generate usable EF images with strong contrast. However, EF imaging together 
with the equation developed by Hatterstrand and Andren had not been applied specifically to fine tertiary $\gamma$ in multi-component, nickel-based superalloys, such as Rene ' 88 DT.

Initially, plasmon imaging was employed. The large beam intensity available from plasmon imaging allowed rapid image acquisition and Fig. 5 shows an example of a plasmon image with some contrast between $\gamma$ and $\gamma$, including the tertiary $\gamma$. Unfortunately, the contrast between the very small tertiary $\gamma$ appeared to diminish rapidly with precipitate size and foil thickness to the extent that the smallest tertiary particles became unobservable below $15 \mathrm{~nm}$.

FTEM Images Using L-edges. A larger number of ionizationedges were available for generating EF images, due to the multiplicity of alloying elements, and a variety of them were used to generate elemental maps and jump ratios images. The strategy for this study was to use the edge that produced EF images with the highest spectra signal-to-noise ration (SNR) and strongest image strength of contrast (CNR). SNRs were calculated from experimentally determined EELS spectra (collected in image-coupled mode) using the equation proposed by Egerton [12] and the CNR for both the jump ratio and elemental maps were calculated by dividing the difference in average pixel intensity between $\gamma$ and $\gamma$ for a given area $(50 \times 50$ pixel size) by the standard deviation of intensity for the same image size. Figs. 6a-j show examples of the elemental maps and jump ratio images of the $\mathrm{L}$-edges from $\mathrm{Cr}, \mathrm{Al}, \mathrm{Cr}$, $\mathrm{Ni}$ and $\mathrm{Ti}$ from
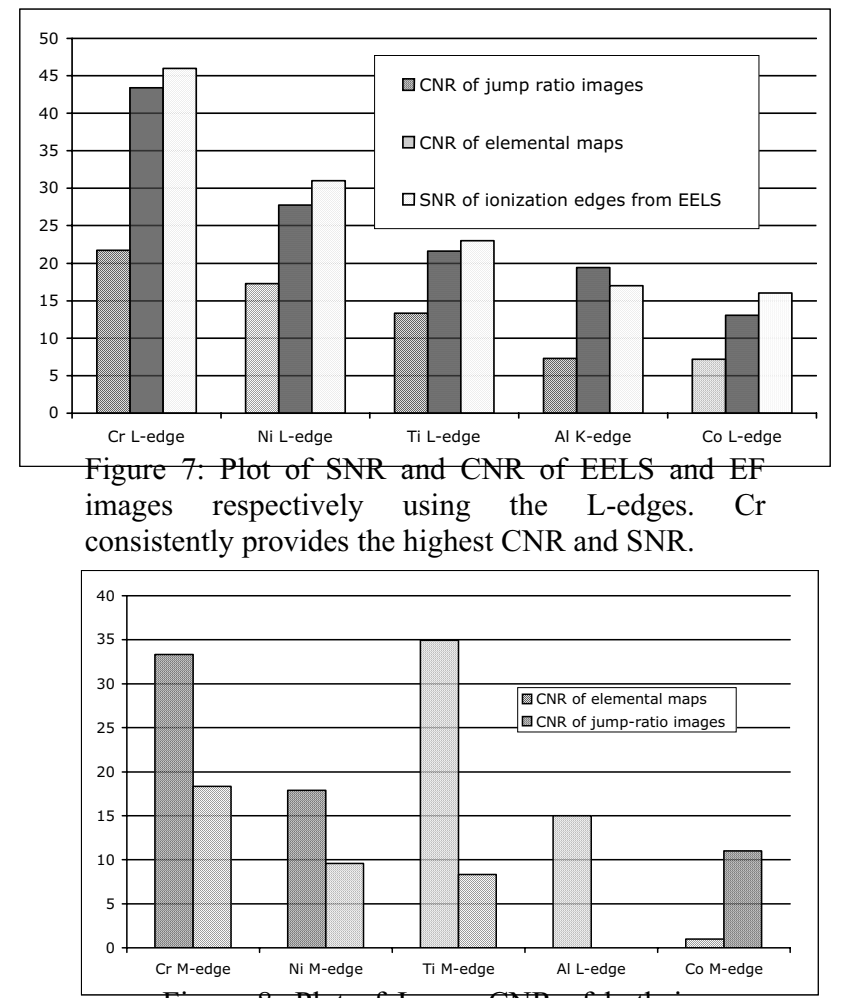

Figure 8: Plot of Image CNR of both jump ratio and elemental maps using the M-edges

which the CNR is obtained. Strong contrast between $\gamma$ and $\gamma$ can be seen in both jump-ratio images and elemental maps although Fig. 7 shows quantitatively that the Cr L-edge offers the highest CNR and SNR from the EF image and EELS spectra, respectively. The jump-ratio images also show that $\mathrm{Cr}$ offers the highest CNR with the same order of degrading CNR from $\mathrm{Cr}$ to Co. However, Fig. 7 also shows that the CNR for jump ratio images are lower than for the elemental maps using the same ionization edges.

EFTEM Images Using M-edges. Elemental maps and jump ratio maps were also acquired in the low-loss region $(30-100 \mathrm{eV})$ although it is important to note that the significant overlap of the M-edges made it difficult to determine accurately which edges contributed to the image contrast for a given energy range (governed by the slit width). Consequently, the elemental maps
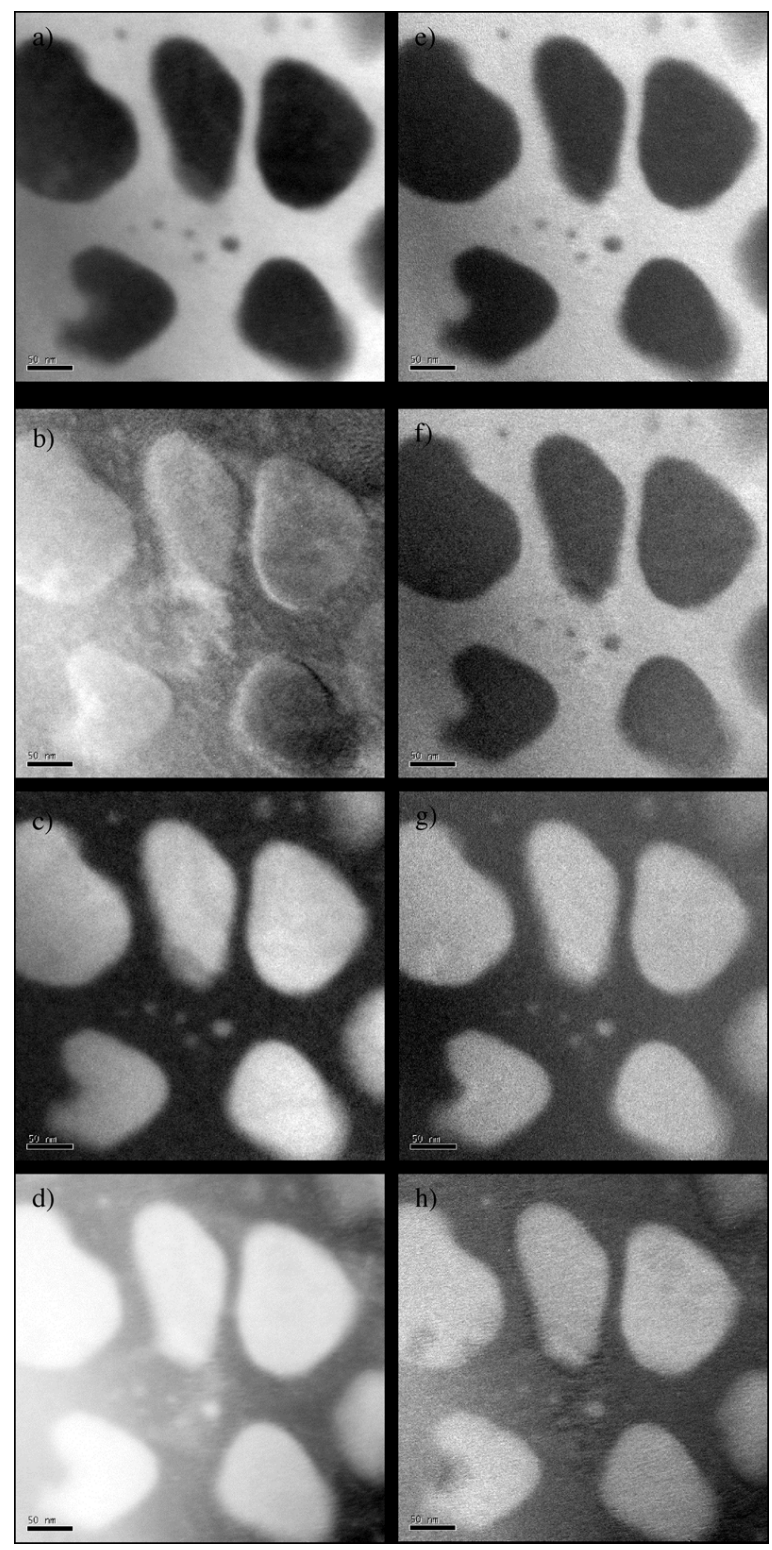

Figures 9a-h: Elemental maps and jump-ratio images using a,e) Cr M-edge b,f) Co M-edge c,g) Ni M-edge and d,h) Ti M-edge

and jump-ratio images were labeled according to the edge for which its threshold energy lies closest to the low-energy side of the post-edge window. No reliable compositional information 
could therefore be obtained from these EF images without complicated deconvolution of the overlapping edges. However, since these images sharply delineate the tertiary $\gamma$ precipitates, they were still considered usable to obtain stereological information required for this study and so the associated CNR for these images were compared with those from the L-edges, despite of the problems mentioned above.

Fig. 8 shows the CNRs from the elemental maps and jump ratio images in Figs. 9a-h using the M-edges of $\mathrm{Cr}, \mathrm{Ni}, \mathrm{Co}$ and $\mathrm{Ti}$. The downward trend in CNR does not follow the same order as that observed for the L-edges, although the Cr edge still possesses the greatest CNR compared with other M-edges, but the CNR for $\mathrm{Cr}$ M-edge was still lower than that for the Cr L-edge).

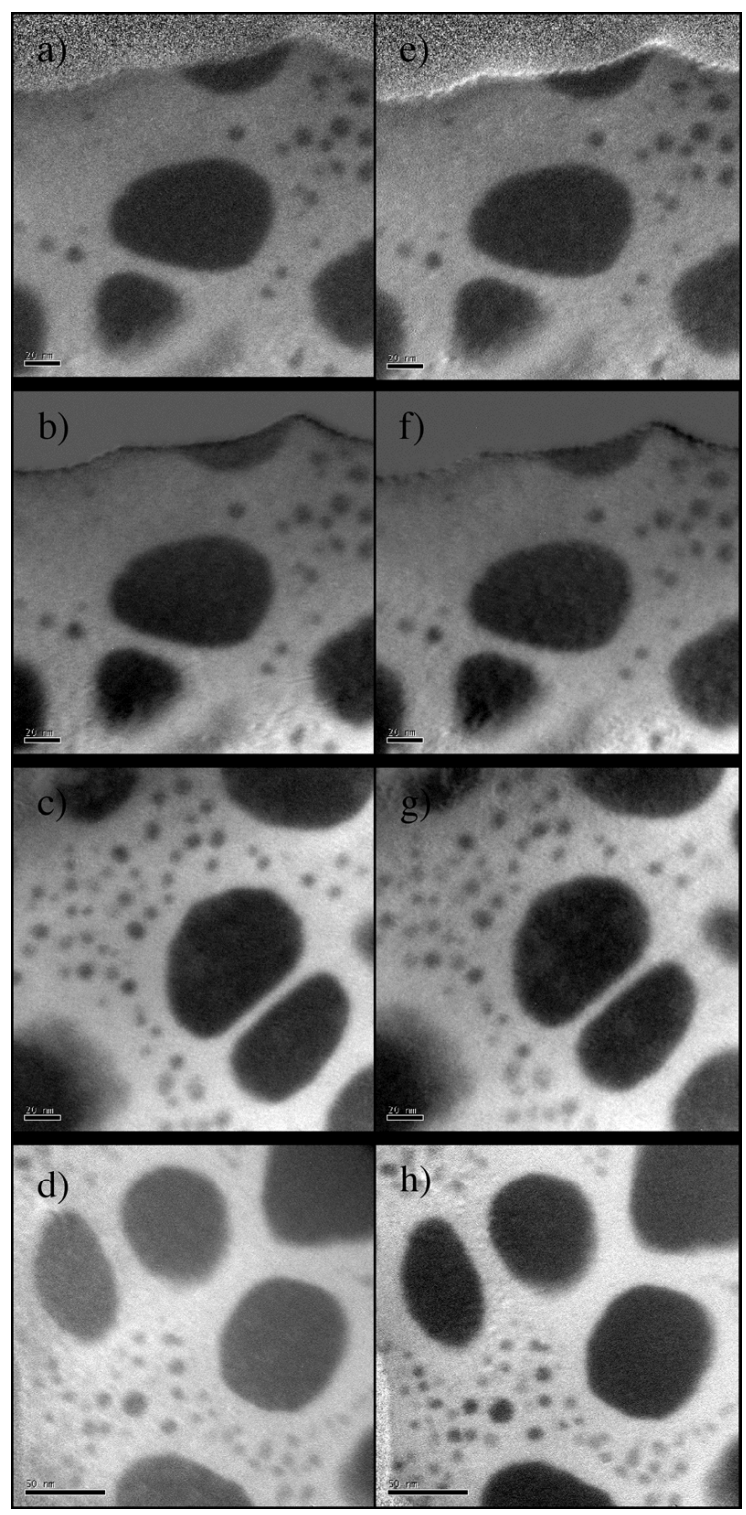

Figure10a-h: Examples of the effects of changing microscope conditions on image quality: Jump ratio images using a) $5 \mathrm{eV}$ and b) $10 \mathrm{eV}$ slit width, Jump-ratio images without objective aperture using c) M-edge and d) L-edge; Jump ratio image e) without aperture f) with aperture
Once the chromium ionization-edge was selected for imaging fine tertiary $\gamma$ precipitates, the parameters for optimal imaging conditions were selected, based on (1) suggestions from other authors [13-16] particularly with regards to post-edge energy position, slit width and collection semi angle $(\beta)$ and (2) other parameters which effect the "quality" of the EF images which were determined in this present study. The initial parameters for generating the optimal imaging conditions are listed in Table ii and their effects on image quality are shown qualitatively in Figs. 10a-f.

Table ii: Optimal microscope and GIF operating conditions for imaging with $\mathrm{Cr}$ ionization- edges.

\begin{tabular}{|l|l|l|}
\hline & L-edge & $\begin{array}{l}\text { M- } \\
\text { edge }\end{array}$ \\
\hline Objective aperture size & $40 \mu \mathrm{m}$ & $20 \mu \mathrm{m}$ \\
\hline Slit width & $40 \mathrm{eV}$ & $5 \mathrm{eV}$ \\
\hline Post-edge position & $600 \mathrm{eV}$ & $57 \mathrm{eV}$ \\
\hline Pre-edge 1 position & $555 \mathrm{eV}$ & $40 \mathrm{eV}$ \\
\hline Pre-edge 2 position & $515 \mathrm{eV}$ & $35 \mathrm{eV}$ \\
\hline Spot size & 1 & 1 \\
\hline Condenser aperture size & $150 \mu \mathrm{m}$ & $150 \mu \mathrm{m}$ \\
\hline
\end{tabular}

\section{Volume Fraction Determination}

EF images were acquired in the thin regions of the TEM foil to maximize image contrast. However, these foil thicknesses were too thin to determine using the Convergent Beam method. Instead, thermal twins present in the microstructure were imaged and measured directly using basic trigonometry to determine the plasmon mean free path $(\lambda)$ and thus calibrate the $t / \lambda$ which is determined from EF thickness maps that were acquired in the same regions as those for the elemental and jump ratio maps.

\section{Discussion}

The ultimate aim of this investigation was to develop a means of acquiring substantial and accurate microstructural information of fine $\gamma$ precipitates (down to $\sim 3 \mathrm{~nm}$ ) in a multi-component nickelbased superalloy. To this end, EF imaging using a $\mathrm{Cr}-\mathrm{L}_{23}$ edge appears to be the optimum method for obtaining this information. Hofer [17] had also acquired the elemental maps and jump-ratio

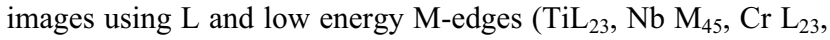
$\mathrm{V} \mathrm{L}_{23}, \mathrm{~V} \mathrm{M}_{23}$ ), but in a niobium alloy to observe very fine carbides and oxides $(3-10 \mathrm{~nm})$. As with the present study, Hofer et al [17] required only microstructural information from the EF images and not quantitative compositional information, and so the jump-ratio method was employed. This approach gave good signal-to-noise ratio (SNR) and strongly reduced the unwanted diffraction contrast that increases undesirably with smaller collection semi-angle (determined by the size of the objective aperture) which is needed to optimize the beam collection efficiency.

Similarly for this present study, the compositional information and absolute intensities within the EF images were of less concern than their imaging characteristics, spatial resolution and the strength of contrast (CNR) between regions of interest $(\gamma / \gamma)$. These parameters are critically important when processing 
images with digital imaging software to measure accurately the relevant microstructural features.

EF images, including those in the work of Hofer [10], are usually acquired using a thermionic $\left(\mathrm{LaB}_{6}\right)$ source because it commonly has an appreciably larger total electron current $(\sim 100$ times larger) which generates higher SNR EF images compared with that of a Field Emission Gun TEM. It is often a trivial matter then, when using a thermionic source, to generate images with high image SNR even in the high energy-loss regimes $\left(\mathrm{L}_{2,3}\right.$-edges above $500 \mathrm{eV}$ ) that usually produce a low beam dose. In this present study, highly converged beams were required to generate sufficient signal when using the L-edges. However, when the beam is highly converged, the decrease in the accelerating voltage necessary when acquiring the two pre-edge images, converges the electron beam further, significantly increasing beam intensity. The overall image intensity of the pre-edge images can then deviate from their true image intensities for the same beam convergence (same operating parameters) introducing extrapolation errors into the background subtraction. Therefore, both $\mathrm{M}$ and L-edges were evaluated in this study.

\section{$\underline{\text { Evaluation of M-edge Images }}$}

A combination of high CNR and superior spatially resolved images can be obtained for the same acquisition times when using M-edges compared with L-edges. This is because the highcount rates available at these energy losses allow imaging parameters to be altered without extensive degradation of image quality. Kothleitner and Hofer [16] show that the theoretical spatial resolution of EF images using M-edges is distinctly less sensitive to slit width than for L-edges. Also, since large signals can be accessed with short acquisition times in this low-loss regime, small slit widths and small collection semi-angles, both of which improve spatial resolution, can be used without significantly degrading the post and pre-edge intensities of the EF images.

Furthermore, since spatial resolution is largely insensitive to slit width, larger slit widths can then be used to increase CNR. It was predicted therefore that the $\mathrm{Cr}-\mathrm{M}$ edge would produce the highest CNR and spatial resolution. However, this assumes that the background intensity in pre-edge regions using the M-edges closely follows the power law function used to extrapolate the post-edge background to determine the core-loss intensities in the final EF image. In fact, the form of the background under and proximate to the M-edges was complex due to both the extensive overlap of low-energy edges from the other elements present in the alloy, and because the intensity fall-off on the high-energy side of the first plasmon peak lies in the same low ionization energy-loss region. These edge overlap factors are not so severe for simple (binary or ternary component) materials when optimizing imaging conditions. However, for the multicomponent aerospace alloy in the present study, these overlap effects can contribute significantly to the degradation and variability of the CNRs compared with EF images using L-edges. Therefore, as well as the extrinsic factors (microscope parameter and filter conditions) which have been well characterized [1317], it is also important to consider the intrinsic sample characteristics in multi-component and multi-phase alloys listed in Table iii when optimizing image CNR
In particular, it appears that edge overlap and absolute concentrations most markedly affect image quality. For instance, high spatial resolution and high CNR using a small slit width can be obtained if much of the core-loss intensity lies in the narrow energy range at the threshold energy, but this maybe negated by the low overall core-loss intensity due to low elemental concentrations present in the alloy. In addition, edge-overlap of the low-energy ionization edges was also of significant concern. Fig. 11 shows the positions and widths of the post and pre-edge windows indicating extensive overlap of combinations of both the post-edge and pre-edge windows of the ionization edges. This complicates further the background extrapolation in the threewindow method. However, there are accidental overlaps of some ionization edges that may be beneficial in generating jump-ratio EF images even though they do not contain quantitative compositional information. Aluminum and nickel, for example, both partition to the $\gamma$ phase and the Al K-edge and Ni M-edge not only lie largely in the same energy loss region, but also possess similar delayed maxima. It may therefore be possible to use the additive effects of both ionization edges in the same postedge window to increase the SNR of a jump-ratio image.

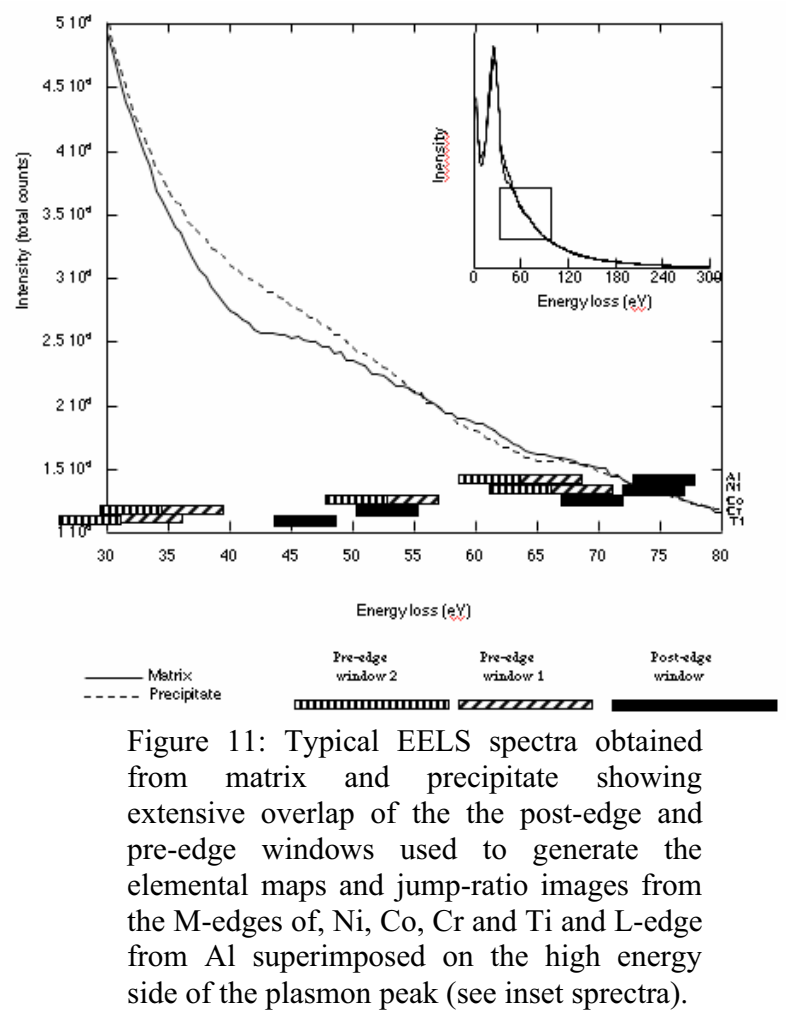

It is also worth noting that the Co M-edge also falls on the Ti Medge but partitioning of these elements occur in $\gamma$ and $\gamma$, respectively. This severely complicates the imaging of these microstructures since the image contrast between $(\gamma$ and $\gamma$ ) from one edge can be offset or inverted by subtly changing the precise positioning of the post and pre-edge windows close to their threshold energy levels. This effect was observed using the standard default settings of the Co M-edge for jump ratio and three-window imaging in which the contrast between the $\gamma$ and $\gamma$ in the elemental map is reversed, as shown in Figs. 9b,f. 
Table iii: List of factors affecting quality of jump ratio images and elemental maps

\begin{tabular}{|l|l|}
\hline Extrinsic Factors & Intrinsic Factors \\
\hline $\begin{array}{l}\text { Condenser } \\
\text { aperture size }\end{array}$ & $\begin{array}{l}\text { Absolute element } \\
\text { concentrations }\end{array}$ \\
\hline $\begin{array}{l}\text { Collection semi- } \\
\text { angle }(\beta)\end{array}$ & $\begin{array}{l}\text { Partitioning strength } \\
\text { between phases }\end{array}$ \\
\hline Slit-width & Edge-overlap and edge-type \\
\hline $\begin{array}{l}\text { Pre and post-edge } \\
\text { positions }\end{array}$ & Foil thickness \\
\hline
\end{tabular}

\section{Determining Precipitate Volume Fraction}

It maybe possible to exploit the reduction in image contrast associated with the mass-fraction effects to estimate $\gamma$ volume fraction without the need to determine the absolute thickness of the TEM foil. This is important because the foil thicknesses used in this study are too thin $(<30 \mathrm{~nm})$ to measure accurately which ultimately determines the accuracy of the estimated volume fraction when using the Hatterstrand and Andren equation.

Both the size of a precipitate and TEM foil thickness affect image contrast if (1) the precipitate is smaller than the throughthickness of the TEM foil and (2) the concentration of the element used for imaging is higher in the matrix. This is because the matrix in the foil, which lies in the same lateral position as the precipitate, projects its higher elemental concentration onto that of the embedded precipitates, reducing the contrast between precipitate and matrix. So, for the same foil thickness, the contrast between an embedded precipitate and matrix should change linearly with the fraction of precipitate that exists through the foil in the direction of the beam. This linear change in contrast with embedded precipitate size can then be used, together with the difference in average intensity between the through-thickness precipitate (no projected matrix contributing to the overall element concentrations of the precipitate) and matrix to determine the volume fraction of the tertiary $\gamma$ precipitates. This assumes that there is negligible compositional difference between the tertiary $\gamma$ and larger through-thickness precipitates.

As an example, Fig. 12 shows an intensity profile across three regions of interest - the matrix, a large $\gamma$ precipitate penetrating through the foil, and a small tertiary $\gamma$ precipitate. The profile across the tertiary $\gamma$ precipitates clearly shows a higher chromium concentration than in the larger $\gamma$ precipitate, indicating that the tertiary precipitate is embedded and therefore smaller than the thickness of the foil. The intensity across the tertiary $\gamma$ precipitate extends to around half that of the larger $\gamma$, suggesting that the precipitate size is half that of the foil thickness. The volume fraction of the tertiary $\gamma$ can then be estimated using the projected areal fraction obtained directly from the EF images and the difference in the strength of contrast of embedded precipitates shown in Fig. 12 using the following equation:

$$
V_{f}=A_{f}^{\text {projected }} * \frac{\left(I_{m}-I_{t}\right)}{\left(I_{m}-I_{s}\right)}
$$

where, $A_{f}$ is the areal fraction, $I_{m}$ is the average image intensity of the matrix, $I_{t}$ is the average intensity of the tertiary $\gamma$ and $I_{S}$ is the average intensity of the through thickness secondary $\gamma$.

It is therefore important to minimize artifact contrast and therefore jump ratio images, which tend to possess less diffraction contrast than elemental maps, should be used to obtain volume fraction information. The accuracy in determining foil thickness and image quality can be developed further using the multi-slice technique [18] under rocking beam illumination [19] to minimize diffraction effects.

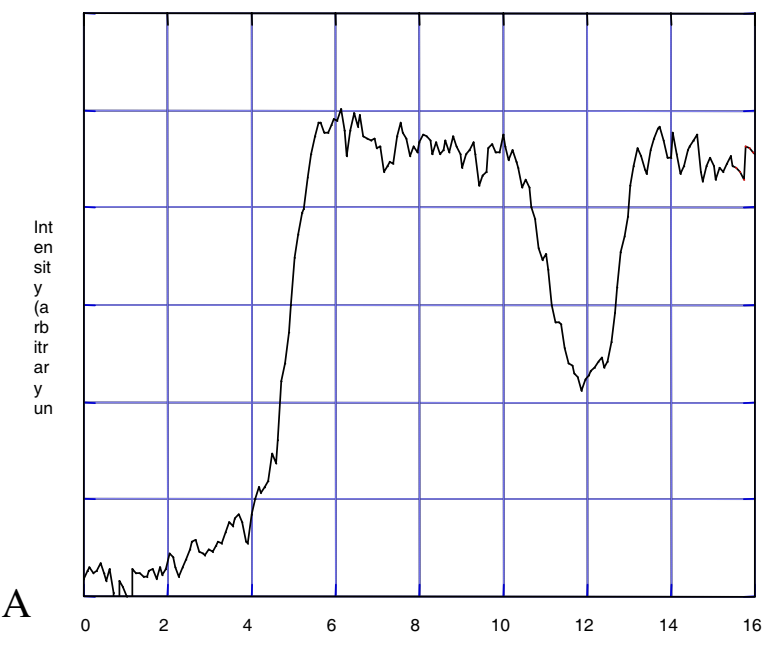

B

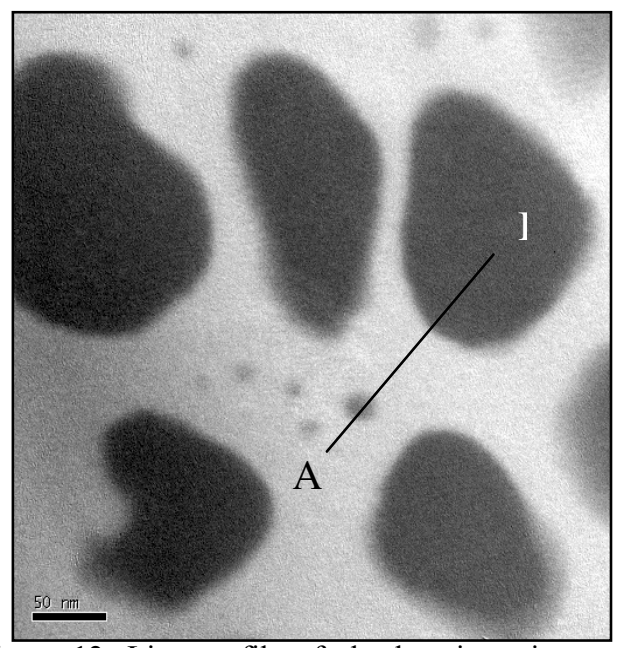

Figure 12: Line profile of absolute intensity across from position $\mathrm{A}$ to $\mathrm{B}$ of an elemental map showing maximum intensity from matrix and mininıum intensity from the large, through thickness secondary $\gamma^{\prime}$ and intermediate intensity across a tertiary $\gamma^{\prime}$ precipitate. The intermediate intensity across the tertiary $\gamma^{\prime}$ suggests that the particle is smaller than the through thickness of the foil whereas the linear fraction of the precipitate through the entire foil thickness can be estimated from the intensity difference between the matrix and the through thickness of the secondary $\gamma^{\prime}$. 


\section{Conclusions}

EFTEM imaging using the Cr L-edge with the jump ratio method is a superior technique for imaging fine $\gamma$ precipitates compared with STEM and CTEM, and may be used on multi-component alloys such as Rene 88 DT.

The volume fraction of tertiary $\gamma$ precipitates embedded in a $\gamma$ matrix may be determined using the intensity of the EF elemental map coupled with the EF thickness maps without requiring the absolute thickness of the TEM foil.

\section{Acknowledgements}

The authors are grateful to the DARPA Accelerated Insertion of Materials (AIM) program and Program Manager Dr. Leo Christodoulou for financial support of this work.

\section{References}

1. D.D. Krueger, R.D. Kissinger and R.D. Menzies, "Development and Introduction of a Damage Tolerant High Temperature Nickel-Base Disk Alloy, Rene' 88 DT"' Superalloys 1992, S.D. Antolovich et al editors, TMS-AIME, Warrendale PA, (1992), 467-476

2. S.T. Wlodeck, M. Kelly and D.A. Aiden, "The structure of Rene 88 DT, "The Structure of Rene' 88 DT", Superalloys 1996, R.D. Kissinger et al editors, TMS-AIME, Warrendale PA, (1996), 129-136

3. P.R. Bhowal, E.F. Wright and E.L. Raymond, "Effects of Cooling rate and Morphoology on Creep and Stress Rupture of a Powder Metallurgy superalloy", Met. Trans. A, 21, (1990), $1709-1717$

4. T. Pollock, Private Communication, 2002

5. S. Sondhi, M. McClean and B. Dyson, "Modeling Creep Deformation of nickel-Base Superalloys in Steady and variable Loading" (8th Progress report on DARPA AIM programme, 2003)

6. A.M. Wusatowska-Sarnek, G. Ghosh, G.B. Olson, M.J. Blackburn and M. Aindow, (2002), in Press
7. M. Aindow, Private Communication, 2002

8. Pratt and Whitney, Private Communication, 2003

9. R.F. Egerton R F, 1996, electron Energy Loss in the Electron Microscope (Plenum: New York), 258

10. F. Hofer and P. Warbichler., "Improved imaging of secondary phases in solids by energy-filtering TEM,"

Ultramicroscopy, 63 (1996), 21-25

11. M. Hatterstrand and H.-O. Andren, "Evaluation of particle size distributions of precipitates in a 9\% chromium steel using energy filtered transmission electron microscopy," Micron, 32 (2001), 789-797

12. R.F. Egerton R F, 1996, electron Energy Loss in the Electron Microscope (Plenum: New York), 123

13. G. Kothleitner and F. Hofer, "Optimization of the Signal to Noise Ratio in EFTEM Elemental Map with Regard to Different Ionization Edge types," Micron, 29 (1998), 349-357

14. O.L. Krivanek, M.K. Kundman and K. Kimoto, Elemental Maps of Materials by Energy-Filtered Imaging," Journal of Microscopy, 180 (1995), 277-287

15. A. Berger and H. Kohl, "Optimum imaging parameters for elemental mapping in an energy filtering transmission electron microscope," Optik, 3 (1993), 159-192

16. T. Pun, J. Ellis and M. Eden, "Optimized acquisition papmeters and statistical detection limit in quantitative EELS," Journal of Microscopy, 135 (1984), 295-316

17. F. Hofer, P. Warbichler and W. Grogger, "Imaging of naometer sized precipitates in solids by electron spectroscopic imaging," Ultramicroscopy, 59 (1995), 15-31

18. P.J. Thomas and P.A. Midgley, "Image spectroscopy I. The advantages of increased spectrral information for compisitional EFTEM analysis," Ultramicroscopy, 88 (2001), 179-186

19. F. Hofer, W. Grogger, G. Kothleitner and P. Warbichler, "Quantitative compositional imaging with energy filtering TEM," Paper presented at EMAG, Sheffield, (1999), 1 